\title{
Atualização em Reumatologia: Artrite Reumatóide Inicial
}

\section{Update in Rheumatology: Early Rheumatoid Arthritis}

\author{
Licia Maria Henrique da Mota ${ }^{(1)}$
}

Nos últimos anos, a generalização do conceito de artrite reumatóide (AR) inicial ou precoce e da existência de janela de oportunidade terapêutica - período no qual a instituição de terapia adequada para a doença acarretaria marcada melhora clínica - firmaram a noção de que diagnóstico e tratamento precoces poderiam modificar o curso da doença.

Concomitantemente, foram desenvolvidos ou aprimorados métodos laboratoriais e de imagem que contribuíram para diagnóstico mais precoce e determinação de prognóstico da $\mathrm{AR}$ inicial, bem como foram instituídas mudanças na forma de abordagem terapêutica da doença, com a utilização de novas classes de drogas.

Não obstante esses avanços, vale ressaltar que o fator limitante à boa resposta terapêutica na AR inicial continua sendo o retardo no diagnóstico e na instituição do tratamento adequado, bem como a dificuldade no manejo da medicação durante o acompanhamento do paciente.

Essa seção traz uma série de artigos recentes que discutem aspectos diagnósticos e terapêuticos da AR inicial.

de Vries-Bouwstra JK, Goekoop-Ruiterman YP, Verpoort KN, Schreuder GM, Ewals JA, Terwiel JP, Ronday HK, Kerstens PJ, Toes RE, de Vries RR, Breedveld FC, Dijkmans BA, Huizinga TW, Allaart CF. Progression of joint damage in early rheumatoid arthritis: association with HLA-DRB1, rheumatoid factor, and anti-citrullinated protein antibodies in relation to different treatment strategies (Progressão do dano articular na artrite reumatóide inicial: associação com HLA-DRB1, fator reumatóide e anticorpos anti-proteínas citrulinadas em relação a diferentes estratégias terapêuticas). Arthritis Reum 58(5):1293-8. Leiden University Medical Center. Leiden, Holanda.

Entre os vários fatores associados à suscetibilidade e à gravidade da artrite reumatóide (AR), listam-se a presença de determinados auto-anticorpos, notadamente o fator reumatóide (FR) e os anticorpos anti-proteínas citrulinadas (ACPA), e a positividade para certos alelos do antígeno leucocitário humano (HLA) de classe II. A presença dos alelos HLA-DRB1 *0101, *0102, *0401, *0404, *0405, ${ }^{*} 0408,{ }^{*} 0410,{ }^{*} 1001$ ou * 1402 , todos compartilhando uma seqüência de aminoácidos na terceira região hipervariável (HVR3), conhecida como epítopo compartilhado (SE), é associada à suscetibilidade à AR e a formas mais destrutivas da doença. Outros estudos demonstram que os alelos DRB1 *0103, *0402, *1102, *1103, *1301, *1302 $\mathrm{e}^{*} 1304$, contendo a seqüência DERAA na HVR3, estão negativamente associados à progressão da $\mathrm{AR}$. O objetivo dos autores foi determinar a associação entre a progressão do dano articular na AR inicial com o HLA-DRBl, o FR e o ACPA.

O estudo foi conduzido utilizando dados do BeSt (Behandelstrategieën voor Reumatoide Artritis), estudo aleatorizado (randomizado), que comparou quatro estratégias terapêuticas, quais sejam, monoterapia seqüencial (grupo 1), terapia com combinação progressiva de drogas (grupo 2), terapia com combinação inicial de metotrexato, sulfassalazina e prednisona (grupo 3 ) e terapia com combinação inicial de metotrexato e infliximabe (grupo 4), em 508 pacientes com AR inicial. O tratamento em todas as estratégias foi conduzido almejando escore de atividade da

Serviço de Reumatologia do Hospital Universitário de Brasília (HUB) da Universidade de Brasília (UnB).

Médica reumatologista do Serviço de Reumatologia do Hospital Universitário de Brasília (HUB) da Universidade de Brasília (UnB), aluna do Programa de Pós-Graduação (Doutorado) em Ciências Médicas da Faculdade de Medicina da Universidade de Brasília (FM-UnB).

Endereço para correspondência: Licia Maria Henrique da Mota, SHIS OI 23 conjunto 02, casa 09, 71660-020, Lago Sul, Brasília, DF, e-mail: licia@unb.br 
doença (DAS) menor ou igual a 2,4. Para fins de análise, considerou-se como progressão da doença o aumento do escore de Sharp/van der Heijde, além da menor mudança detectável $(4,6)$, ao longo de dois anos. Aplicou-se análise de regressão logística multivariada para predizer a progressão da doença de acordo com a presença ou a ausência do SE, seqüência DERAA, FR e ACPA, com correção para outras características de base.

Como resultado, encontrou-se que a presença do SE não previa a ocorrência de doença progressiva: o odds ratio nos grupos 1, 2, 3 e 4, respectivamente, foi de 1,4 , 2,6, 1,9 e 3,0. Os pacientes que apresentavam a seqüência DERAA não estavam protegidos contra a progressão da doença (odds ratio 0,4, $1,4,0,9$ e 0,9 , nos grupos $1,23 \mathrm{e}$ 4 , respectivamente). Já a positividade para FR e ACPA foi capaz de predizer a ocorrência de doença progressiva no grupo 1 (odds ratio 4,7 - intervalo de confiança - IC-95\%: 1,5-14,5) para o FR e 12,6 (IC95\%: 3,0-51,9) para ACPA, mas não nos grupos 2 a 4 . Para o FR, odds ratio (IC95\%) de $1,5(0,5$ a 4,9), 1,0 (0,3-3,3) e 1,4 (0,4-4,8) nos grupos 2, 3 e 4, respectivamente. Para ACPA, odds ratio (IC95\%) de $3,4(0,8-14,2), 1,7(0,5-5,4)$ e $1,8(0,5-6,8)$ nos grupos 2,3 e 4 , respectivamente.

Em conclusão, nos pacientes com AR inicial tratados com o objetivo de controle estrito do DAS, não se observou associação significativa entre o status do HLA-DRBl e a progressão radiográfica. O FR e os ACPA foram preditores de doença progressiva apenas nos pacientes tratados com monoterapia seqüencial. Essas observações sugerem que o tratamento efetivo pode prevenir a evolução da progressão radiográfica, mesmo em pacientes com fatores de risco para dano grave.

Innala L, Kokkonen H, Eriksson C, Jidell E, Berglin E, Dahlqvst SR. Antibodies against mutated citrullinated vimentin are a better predictor of disease activity at 24 months in early rheumatoid arthritis than antibodies against cyclic citrullinated peptides (Anticorpos antivimentina citrulinada mutada são um melhor preditor de atividade da doença aos 24 meses que anticorpos antipeptídeos citrulinados cíclicos, na artrite reumatóide inicial). J Rheumatol 35(6):1002-8, 2008. Departamentos de Reumatologia e Imunologia Clínica do Hospital Universitário de Umeå. Umeå, Suécia.

A detecção dos anticorpos contra peptídeos/proteínas citrulinadas (ACPA) tem-se tornado ferramenta valiosa no diagnóstico da AR e na predição do seu prognóstico clínico. Distinguir pacientes com doença inicial que desenvolverão forma leve da doença, com bom prognóstico, daqueles com forma grave e prognóstico restrito, poderia permitir a individualização e a otimização da terapêutica.

Há pouco tempo, foram descritos os anticorpos contra vimentina citrulinada mutada (anti-MCV) como evolução do protocolo para detecção de anticorpos contra a vimentina naturalmente citrulinada (anti-Sa), altamente específicos para AR. Demonstrou-se que a vimentina, encontrada nas células sinoviais da AR, é secretada e modificada por macrófagos, na dependência de sinais pró-inflamatórios, o que faz dessa proteína um auto-antígeno interessante para a avaliação da AR. Tem-se proposto que os anticorpos anti-MCV seriam melhores marcadores prognósticos para AR do que os anti-CCP.

Por outro lado, a associação entre o HLA-DRBl e a AR é bem documentada, e recentemente um gene fora da região HLA, o PTPN22 C1858T, também tem sido associado à doença.
O objetivo dos autores foi avaliar os valores preditivos para progressão de doença de vários ACPA e sua relação com o polimorfismo dos alelos PTN22 1858 C/T e HLADRBI na AR inicial. Entre os ACPA, foram estudados os anticorpos anti-MCV e anti-CCP 2 e 3 (ambos do isotipo $\mathrm{IgG}$ ) e 3.1 (isotipos $\operatorname{IgG}$ e IgA).

Esses ACPA foram analisados na avaliação inicial de 210 pacientes com AR inicial e 102 indivíduos da população controle, utilizando enzima imunoensaio (ELISA). Para cada anticorpo, foi construída uma curva ROC. A atividade da doença (articulações dolorosas e inflamadas, escala visual analógica para saúde global, DAS28, velocidade de hemossedimentação - VHS- e proteína C reativa) foi verificada na avaliação basal e regularmente por 24 meses. Foram também realizadas radiografias de mãos e pés, graduadas pelo escore de Larsen.

Como resultado, observou-se que pacientes com anticorpos anti-MCV apresentaram redução significativamente menor no DAS28 ao longo do tempo $(\mathrm{p}<0,01)$, e tiveram a área sob a curva significativamente aumentada para DAS28 $(\mathrm{p}<0,05)$, VHS $(<0,01)$ e proteína C reativa $(\mathrm{p}<0,01)$, comparados com aqueles anti-MCV negativos. Diferenças correspondentes não foram encontradas em pacientes com anticorpos anti-CCP2, CCP3 o CCP3.1. A progressão 
radiográfica $(\mathrm{p}<0,0001-0,01)$ e o prognóstico radiológico $(\mathrm{p}<0,0001-0,01)$ aos 24 meses foram significativamente preditos por todos os ACPA após ajustes da linha de base. A variante PTPN22 T e os alelos HLA-DRBl não se correlacionaram à progressão radiológica ou à atividade inflamatória ao longo do tempo.
Em conclusão, os anticorpos anti-MCV se associaram à AR mais grave, conforme avaliação pelo DAS28, VHS e contagem de articulações inflamadas ao longo do tempo, em comparação aos anticorpos anti-CCP2, CCP3 o CCP3.1. A progressão radiográfica foi igualmente prevista pelos quatro anticorpos.

Liao KP, Batra KL, Chibnik L, Schur PH, Costenbader KH. Anti-CCP revised criteria for the classification of rheumatoid arthritis (Critérios revisados com anti-CCP para a classificação da artrite reumatóide). Ann Rheum Dis 67(11): 1557-61, 2008. Divisão de Reumatologia, Alergia e Imunologia e Departamento de Medicina, Hospital Brigham. Boston, Estados Unidos.

Os critérios classificatórios para AR do Colégio Americano de Reumatologia (ACR) foram desenvolvidos inicialmente em 1957, e revisados em 1987, com base em indivíduos com AR de longa duração (média de 7,7 anos de sintomas), e são o padrão atual para a seleção de pacientes para estudos clínicos.

Tais critérios apresentam sensibilidade de $91 \%$ a $94 \%$ e especificidade de $89 \%$ para AR estabelecida. No entanto, eles incluem características raras na AR de início recente, como alterações radiográficas (erosões) e nódulos reumatóides, sendo considerados subótimos para a identificação de indivíduos com AR inicial (sensibilidade de $40 \%$ a $90 \%$ e especificidade de $50 \%$ a $90 \%$ ).

Outrossim, na última década, testes laboratoriais mais sensíveis e específicos para a identificação da AR inicial têm sido desenvolvidos, em especial os anticorpos anti-CCP. Na artrite inflamatória inicial, a especificidade dos anti-CCP para a detecção da AR varia de $90 \%$ a $97 \%$, em comparação ao FR ( $80 \%$ a $90 \%)$. Os anticorpos anti-CCP são amplamente utilizados para o diagnóstico da AR inicial, mas não estão incluídos nos critérios do ACR 1987.

Os autores desenvolveram e testaram a performance de novos critérios classificatórios para $\mathrm{AR}$, incorporando o anti-CCP. Como método, foram identificados todos os indivíduos vistos no Centro de Artrite do Hospital Brigham com FR e anti-CCP testados simultaneamente, entre $1^{\circ}$ de janeiro e 30 de junho de 2004, e revisados os registros médicos quanto aos critérios do ACR, diagnóstico reumatológico, FR e anti-CCP.

Os critérios do ACR foram revisados de três formas: (1) adicionando o anti-CCP (critérios do ACR 1987 + antiCCP), (2) substituindo o critério nódulos reumatóides pelo critério anti-CCP (CCP 7 critérios) e (3) substituindo os critérios nódulos reumatóides e erosões radiográficas pelo critério anti-CCP (CCP 6 critérios).

Foram comparadas sensibilidade e especificidade para os diversos critérios, em todos os indivíduos e naqueles que apresentavam sintomas compatíveis com artrite por menos de seis meses.

Foram analisados os registros médicos de 292 indivíduos, com média de idade de 54 anos, predominando o gênero feminino (82\%) e média de sintomas de 4,1 anos. Dezessete por cento tinham FR positivo e $14 \%$ anti-CCP positivo, na avaliação inicial. Setenta e oito pacientes $(27 \%)$ tiveram o diagnóstico de AR feito pelo reumatologista.

Comparado ao diagnóstico do reumatologista na avaliação mais recente (em média nove meses após a consulta de base, quando o FR e anti-CCP foram verificados), os critérios do ACR de 1987 tiveram sensibilidade de 51\% e especificidade de $91 \%$ para a classificação da $\mathrm{AR}$, considerando-se todos os indivíduos. $\mathrm{O}$ acréscimo do anti-CCP aos critérios existentes resultou melhora da sensibilidade de $51 \%$ para $55 \%$, sem mudança da especificidade.

Os critérios com CCP (6 ou 7 critérios), removendo nódulos reumatóides ou nódulos reumatóides e erosões radiográficas, aumentaram a sensibilidade comparados aos critérios do ACR de 1987 para todos os indivíduos (77\% e $74 \%$, respectivamente, comparados a $51 \%$ dos critérios clássicos), bem como para a classificação de indivíduos com sintomas iniciais ou de longa duração. Para todos os pacientes, os novos critérios revisados ocasionaram decréscimo da especificidade para 70\% a 80\%, em comparação aos critérios do ACR de 1987.

O CCP 6 critérios, em relação aos critérios clássicos de 1987, aumentou a sensibilidade em indivíduos com sintomas há menos de seis meses: $63 \%$ versus $25 \%$, com perda da especificidade ( $72 \%$ versus $86 \%$ ). 
Concluiu-se que o CCP 6 critérios, em especial, melhorou a sensibilidade dos critérios do ACR de 1987, de maneira mais marcada para indivíduos com menos de seis meses de sintomas, e poderia ser utilizado para a classificação de pacientes com AR em estudos clínicos.

Hetland ML, Ejbjerg BJ, Hørslev-Petersen K, Jacobsen S, Vestergaard A, Jurik AG, Stengaard-Pedersen K, Junker P, Lottenburger T, Hansen I, Andersen LS, Tarp U, Skjødt H, Pedersen JK, Majgaard O, Svendsen AJ, Ellingsen T, Lindegaard HM, Christensen AF, Vallø J, Torfing T, Narvestad E, Thomsen HS, Ostergaard M. MRI bone oedema is the strongest predictor of subsequent radiographic progression in early rheumatoid arthritis. Results from a 2 year randomized controlled trial (CIMESTRA) (Edema ósseo à ressonância magnética é o preditor mais forte de subseqüente progressão radiográfica na artrite reumatóide inicial. Resultados de um estudo controlado aleatorizado de 2 anos - CIMESTRA). Ann Rheum Dis 2008, publicado on line em 3 de abril de 2008, doi: 10.1136/ard.2008.088245. Hospital do Reumatismo, Universidade do Sul da Dinamarca. Gråsten, Dinamarca.

A presença de edema medular ósseo à imagem de ressonância magnética (IRM) no início da AR tem sido relacionada à progressão do dano articular após um a seis anos. Há poucos estudos sobre a importância relativa de outros novos potenciais fatores preditivos, como o antiCCP, em comparação ao edema ósseo à IRM.

O objetivo do estudo foi investigar se alterações precoces à IRM teriam valor prognóstico para o desenvolvimento de erosões à radiografia convencional. Outros potenciais marcadores prognósticos foram examinados para identificar preditores independentes de progressão radiológica durante o acompanhamento de dois anos em um estudo clínico aleatorizado, duplo-cego, de pacientes com AR inicial (CIMESTRA).

Cento e trinta pacientes com AR inicial foram tratados com a finalidade de alcançar bom controle da doença (segundo DAS28), com drogas convencionais modificadoras do curso da doença (DMARDs). Foram utilizadas doses escalonadas de metotrexato $(7,5 \mathrm{a} 20 \mathrm{mg} / \mathrm{semana})$ e placebo/ciclosporina $(2,5 \mathrm{a} 4 \mathrm{mg} / \mathrm{kg})$ ou metotrexato $(7,5$ a $20 \mathrm{mg} /$ semana) em combinação com ciclosporina $(2,5$ a $4 \mathrm{mg} / \mathrm{kg}$ ). As articulações edemaciadas eram tratadas com infiltração intra-articular de betametasona. Durante o segundo ano, a ciclosporina (ou o placebo-ciclosporina) era retirada gradativamente, sendo adicionada hidroxicloroquina a todos os pacientes. $\mathrm{O}$ metotrexato e as injeções intra-articulares de betametasona eram mantidos.

Foram realizados na avaliação inicial: IRM dos punhos $(\mathrm{n}=130)$ ou de punhos e metacarpofalangeanas (MCF) $(\mathrm{n}=89)$ (OMERACT RAMRIS), radiografias das mãos, punhos e pés (escore de Sharp/van der Heijde-TSS), escore de atividade da doença (DAS28), anti-CCP, SE e status quanto ao tabagismo. Aplicada análise de regressão múlti- pla, com delta-TSS (0-2 anos) como variável dependente, e, como variáveis explanatórias, DAS28 da avaliação inicial, TSS, escore de edema ósseo, sinovite ou erosão à IRM, anti-CCP, tabagismo, SE, idade e gênero.

$\mathrm{Na}$ avaliação basal, observou-se DAS28 de 5,6 (2,4 a $8,0)$, anti-CCP positivo em $61 \%$ dos casos e erosões radiográficas em 56\%. Aos dois anos de acompanhamento, o DAS28 médio foi de $2,0(0,5$ a 5,7$), 56 \%$ dos pacientes atingiram remissão segundo o DAS e $26 \%$ apresentaram progressão radiográfica (semelhante para o grupo punho versus punho + MCF). O escore de edema à IRM foi o único preditor independente de delta -TSS. No grupo punho + MCF, o coeficiente foi de 0,75 (IC95\%: 0,56-0,97), $\mathrm{p}<0,00001$. No grupo que realizou IRM de punho apenas, o coeficiente foi de 0,56 (0,4l-0,77), p < 0,00001. O escore do edema ósseo explicou $41 \%$ da variação na progressão do TSS (grupo punho + MCF) e 25\% no grupo que realizou exame apenas do punho (Pearson $r=0,64 \mathrm{e}$ $r=0,50$, respectivamente).

Em um ensaio aleatorizado controlado almejando remissão em pacientes com $\mathrm{AR}$ precoce, a conclusão desse estudo foi que o escore de edema ósseo RAMRIS à IRM das MCF e punhos (e dos punhos, isoladamente) foi o mais forte preditor independente de progressão radiográfica em mãos, punhos e pés, após dois anos de acompanhamento. O escore de sinovite e erosão à IR, bem como o DAS28, anti-CCP, SE, tabagismo, idade e gênero não foram preditores independentes de risco nesse estudo.

A interpretação dos dados sugere que a IRM poderia ser ferramenta útil, suplementar aos exames convencionais utilizados na avaliação de pacientes com AR inicial, com a finalidade de otimizar a identificação de pacientes com alto risco de progressão para formas erosivas. 
Hafström I, Albertsson K, Boonen A, van der Heijde DM, Landewé R, Svensson B. Remission achieved after 2 years treatment with low dose prednisolone in addition to DMARDs in early $R A$ is associated with reduced joint destruction still present after 4 years: an open two year continuation study (Remissão alcançada após dois anos de tratamento com baixas doses de prednisolona em associação à DMARDs na artrite reumatóide inicial é associada com redução da destruição articular ainda presente após quatro anos: continuação de um estudo aberto de dois anos). Ann Rheum Dis 2008, publicado on line em 17 de abril de 2008, doi:10.1136/ard.2008.087833. Departamento de Reumatologia, Hospital da Universidade de Karolinska. Estocolmo, Suécia.

O objetivo atual do tratamento da AR é induzir a remissão clínica, fundamentando-se na hipótese de que o processo inflamatório leva ao dano estrutural. No entanto, induzir a remissão é, em muitos casos, difícil. A fim de melhorar a resposta ao tratamento, diversas estratégias terapêuticas têm sido empregadas.

Nos últimos anos, tem-se proposto que os glicocorticóides em baixas doses, na AR inicial, teriam potencial de droga modificadora da doença, bem como a habilidade de induzir remissão. No entanto, não é certo se a terapia continuada com baixas doses de corticoesteróides, ou o uso por um período inicial restrito, teriam benefícios a longo prazo.

O intuito dos autores foi avaliar se a remissão induzida por baixas doses de prednisolona, somadas à primeira DMARD durante os dois anos iniciais de acompanhamento de pacientes com AR inicial, teria efeito mantido sobre o dano radiológico e a capacidade física, medidos pelo período de quatro anos.

Foram utilizados dados do estudo BARFORT sobre o uso de glicocorticóides, referentes a 150 do total de 211 pacientes elegíveis com $\mathrm{AR}$, que haviam sido aleatorizados para o uso (grupo P) ou não-uso (grupo não $\mathrm{P}$ ) de 7,5 $\mathrm{mg}$ de prednisolona, associada à DMARD inicialmente utilizada. Foram realizadas radiografias de mãos e punhos, avaliadas pelo escore de Sharp/van der Heijde. A remissão foi definida por um DAS28 menor que 2,6. A capacidade funcional foi avaliada pelo Health Assessment Questionarie (HAQ) e a densidade mineral óssea medida por DEXA.
A idade média dos pacientes foi de 53 anos (grupo P) e 57 anos (grupo não $\mathrm{P}$ ). Dos indivíduos avaliados, 64\% eram mulheres e $64 \%$ tinham FR positivo. A duração da doença à avaliação inicial foi de seis meses. Após dois anos, a proporção de pacientes em remissão foi de $\mathbf{5 5 \%}$ (grupo $\mathrm{P}$ ) versus $30 \%$ (grupo não $\mathrm{P}$ ), $\mathrm{p}=0,003$. A análise longitudinal demonstrou que, durante todo o curso da doença, os pacientes em uso de prednisolona tiveram probabilidade maior de atingir remissão.

Os pacientes que estavam em remissão após dois anos de acompanhamento, comparados àqueles que não atingiram remissão, apresentavam escore de Sharp total, escore de erosão e escore de redução do espaço articular mais baixos, no segundo e quarto anos. Os pacientes em remissão após dois anos apresentavam HAQ significativamente menor que os demais, independente do tratamento ou não com esteróides.

As mudanças na densidade mineral óssea durante os quatro anos não diferiram entre aqueles que atingiram ou não remissão e foram semelhantes entre os dois grupos de tratamento.

A conclusão dos autores foi que a prescrição de 7,5 mg de prednisolona diariamente, em associação à DMARD, aumenta a taxa de remissão em pacientes com AR inicial, o que parece ter efeito benéfico e sustentado sobre o dano radiológico. Os autores sugerem que a remissão devam ser o alvo do tratamento da AR, e que baixas doses de esteróides possam ser usadas, em associação à DMARD tradicionais, no tratamento da AR inicial.

Choy EH, Smith CM, Farewell V, Walker D, Hassell A, Chau L, Scott DL. Factorial randomised controlled trial of glucocorticoids and combination disease modifying drugs in early rheumatoid arthritis (Estudo controlado randomizado fatorial de glicocorticóides e combinação com drogas modificadoras do curso da doença na artrite reumatóide inicial). Ann Rheum Dis 67(5):656-63, 2008. King's College School of Medicine, Weston Education Centre. Londres, Inglaterra.

O tratamento da AR inicial com DMARD em monoterapia alcança resultados muitas vezes incompletos, de maneira que a terapêutica intensiva parece preferível. No entanto, os benefícios relativos de combinar duas DMARDs, uma DMARD com glicocorticóide ou as duas DMARD com glicocorticóide são incertos. Os autores avaliaram as terapêuticas propostas em um estudo controlado aleatorizado com desenho fatorial. 
Os estudos fatoriais geralmente permitem avaliação simultânea de duas intervenções. Os pacientes são aleatoriamente direcionados para um de quatro grupos, com cruzamento de placebos, de modo que o efeito de cada intervenção possa ser comparado simultaneamente, e as interações sinérgicas ou negativas entre as intervenções possam ser avaliadas.

Foi realizado estudo fatorial aleatorizado duplo-cego em pacientes com AR com no máximo dois anos de diagnósti$\mathrm{co}$, tratados com metotrexato. Avaliaram-se os benefícios da adição de ciclosporina, nove meses de tratamento intensivo com prednisolona ou ambas (terapia tríplice). O desfecho primário analisado foi o número de pacientes com novas erosões. Os desfechos secundários considerados foram o escore de Larsen, a capacidade física, a qualidade de vida e a ocorrência de efeitos adversos.

Foram avaliados 1.391 pacientes, dos quais 467 foram selecionados. Os pacientes foram divididos nos seguintes grupos: (a) braço aberto com metotrexato (iniciando com $7,5 \mathrm{mg} / \mathrm{semana}$, com aumento subseqüente até a dose-alvo de $15 \mathrm{mg} / \mathrm{semana}$ ); (b) step-down: prednisolona associada ao metotrexato (dose de prednisolona inicial de $60 \mathrm{mg} / \mathrm{dia}$, com redução para 7,5 mg em 6 semanas, 7,5 mg/dia da sexta à vigésima oitava semana, e interrupção na trigésima quarta semana); e (c) ciclosporina, iniciada três meses após metotrexato (dose inicial de $100 \mathrm{mg} /$ dia, com aumento gradual até a dose alvo de $3 \mathrm{mg} / \mathrm{kg}$ ).
Durante os dois anos de acompanhamento, 132 pacientes $(28 \%)$ tiveram mudança da terapêutica e 88 (19\%) perderam o seguimento. O número de pacientes com novas erosões foi reduzido em cerca de $50 \%$ pela adição de ciclosporina ou prednisolona $(\mathrm{p}=0,01$ e $\mathrm{p}=0,03)$; ambos os tratamentos reduziram aumentos nos escores de Larsen em mais de duas unidades ( $\mathrm{p}=0,008 \mathrm{e} \mathrm{p}=0,003)$. Uma redução adicional nas erosões foi vista com a combinação de ambos os tratamentos, porquanto seus efeitos sobre o prognóstico radiográfico ocorreram de maneira independente.

A terapia tríplice reduziu a incapacidade e melhorou a qualidade de vida, em comparação ao metotrexato, e ciclosporina e prednisolona apresentaram ação sinérgica. Entre aqueles com terapia tríplice, um número maior de pacientes abandonou a terapêutica por eventos adversos, mas não houve aumento na ocorrência de eventos adversos sérios.

Os autores concluíram que os resultados do estudo permitem afirmar que de fato existe janela de oportunidade em pacientes com AR inicial, quando uma combinação terapêutica intensiva pode produzir benefícios sustentados sobre o dano articular e a incapacidade. Nesse estudo, embora as combinações de metotrexato-prednisolona tenham reduzido a ocorrência de erosões, foi observado que o efeito sinérgico de duas DMARDs é necessário para melhorar a qualidade de vida. 